\title{
Manufacture of 3D microfiber coil resonators
}

\author{
Fei Xu and Gilberto Brambilla \\ Optoelectronics Research Centre, University of Southampton, Southampton, SO17 1BJ, UK
}

\begin{abstract}
D microfiber coil resonators are experimentally demonstrated. Three-turn and four-turn microcoil resonators are fabricated by wrapping a microfiber on a low refractive index rod. The extinction ratio and free spectrum range are $\sim 10 \mathrm{~dB}$ and $\sim 1 \mathrm{~nm}$ respectively.
\end{abstract}

\section{Introduction}

High-Q microresonators based on sub-wavelength microfibers have attracted much attention in the recent years because of the enormous progress in the fabrication of low-loss submicrometric optical wires, which allow for low-loss evanescent wave guiding [1-4]. Microfiber coil resonators (MCR) boast an extensive variety of promising applications such as slow light generation, optical filtering, optical signal processing and sensing. Two types of onedimension MCR in air have been reported in previous publications: the self-touching loop resonator [5-7] and the knot resonator [1, 8]. A 3D multi-turn MCR was suggested in reference 9 this type of MCR is more flexible and can be used to manufacture ultra high Q-factor resonators and high sensitivity microchannel sensor [10]. Although the experimental demonstration of a MCR was recently reported for a microfiber immersed in a refractive index matching liquid [11], the extinction ratio of such a device was smaller than $1.5 \mathrm{~dB}$. In this paper we experimentally demonstrated for the first time to our knowledge a three-turn and four-turn MCRs by manually wrapping a microfiber on a low refractive index rod. It has a simple manufacturing process and it is stable in air.

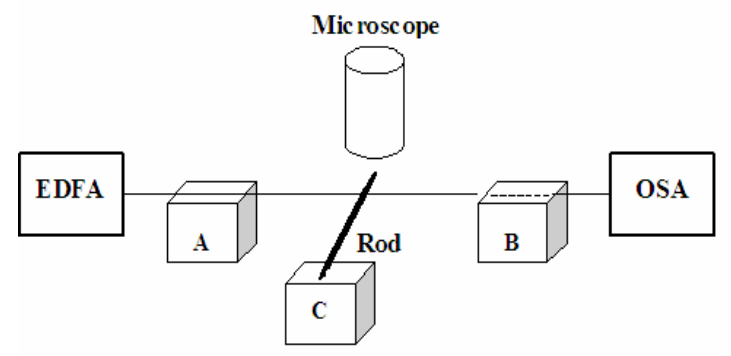

Fig. 1 the set up used to manufacture 3D MCR. A, B and $C$ are $X Y Z$ stages.

\section{Fabrication}

The microfiber used in the experiment was fabricated using the set-up presented in reference 12 and a microheater (NTT-AT, Japan). The microfiber radius and the length of the uniform waist region were $\sim 1.5 \mu \mathrm{m}$ and $\sim 4 \mathrm{~mm}$ respectivetily.

The 3D MCR was manufactured with the set up shown in Fig. 1. At first, the microfiber had its pigtails connected to an Erbium-doped fiber amplifier (EDFA) and an optical spectrum analyzer (OSA) to check, in real time, the resonator properties during fabrication; then, with the aid of a microscope, the microfiber was wrapped on a low refractive index rod while one of its ends was fixed on a 3D stage; finally the other microfiber end was fixed to another $3 D$ stage and both microfiber ends were tuned to find the optimum resonator spectrum. This methodology is similar to that theoretically predicted for the design optimization of a 3D microcoil resonators presented in literature $[13,14]$. Because the coupling coefficient between two adjacent microfiber coils is weak, the microfibre coils need to be kept as close as possible.

In these experiments, the microfiber was wrapped on a low refractive index rod to maximise the MCR temporal stability and robustness. By using this process losses can be significant because of microbends and confinement losses. If the loss is too high, it is difficult to optimise the microfiber position and MCR geometry. Generally, the loss can be minimised by increasing the microfiber thickness and the rod diameter, by using a low refractive index material for the rod and by improving the rod surface smoothness. In these experiments a rod with total diameter D 560 $\mu \mathrm{m}$ was coated with Teflon @AF (DuPont, United States), to provide a low refractive index $(n \sim 1.3$ at $\sim 1.55 \mu \mathrm{m})$ at the interface with the microfiber.

\section{Geometry and spectrum}

Figure 2 shows the pictures of a three-turn and four turn MCR. The manual fabrication of the MCR implies that pitches between adjacent turns are not uniform, and the microfibres present some degree of twist. Figure 3 shows the resonator spectrums of the two MCRs. The dashed line is the reference spectrum when the microfiber and the rod are not in contact. The spectra show a complicated profile because the coupling among the three or four turns is irregular and not uniform.

The maximum extinction ratios are $10 \mathrm{~dB}$ for thereturn MCR and $9.5 \mathrm{~dB}$ for the four-turn MCR. The spectrum of the three-turn MCR can be simplified as the combination of two spectra of simple ring resonators, one is dominant $(10 \mathrm{~dB})$ while the other one is weak $(3 \mathrm{~dB})$; the free spectral ranges (FSR) 
of the two MCRs are the same (about $0.9 \mathrm{~nm}$ ); The four-turn MCR is more complicated, and can be possibly assumed as the superposition of three simple ring resonators with different FSR: the weak one has FSR $\sim 0.93 \mathrm{~nm}$ and $3 \mathrm{~dB}$ extinction, the intermediate one a FSR 1.85 and a $5 \mathrm{~dB}$ extinction and the strongest one a FSR 1.85 nm and a $9.5 \mathrm{~dB}$ extinction ratio.

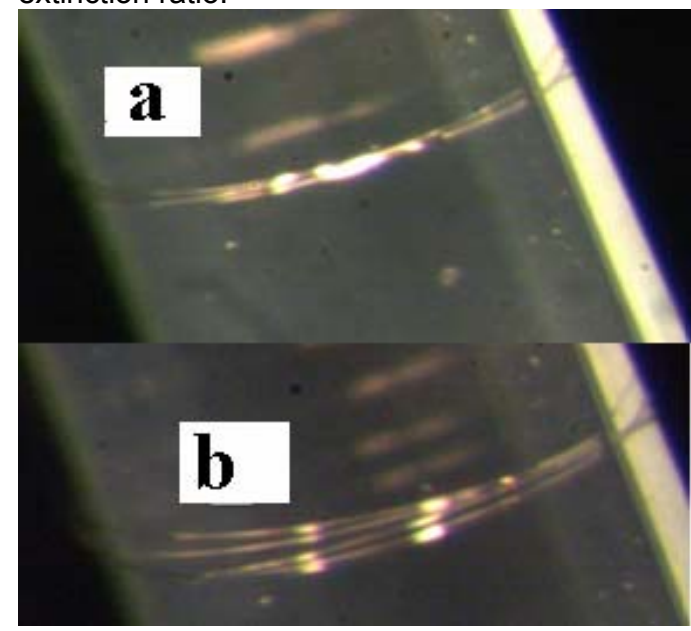

Fig. 2 Pictures of 3D MCRs. The number of turns in the MCR is three in (a) and four in (b).

The loss induced by the wrapping of the microfiber on the rod was $2.5 \mathrm{~dB}$ for three-turn MCR and $5 \mathrm{~dB}$ for the four-turn MCR. The loss is due to surface roughness, refractive index discontinuity at the input/output touching points, and leaky modes associated to the rod refractive index profile.

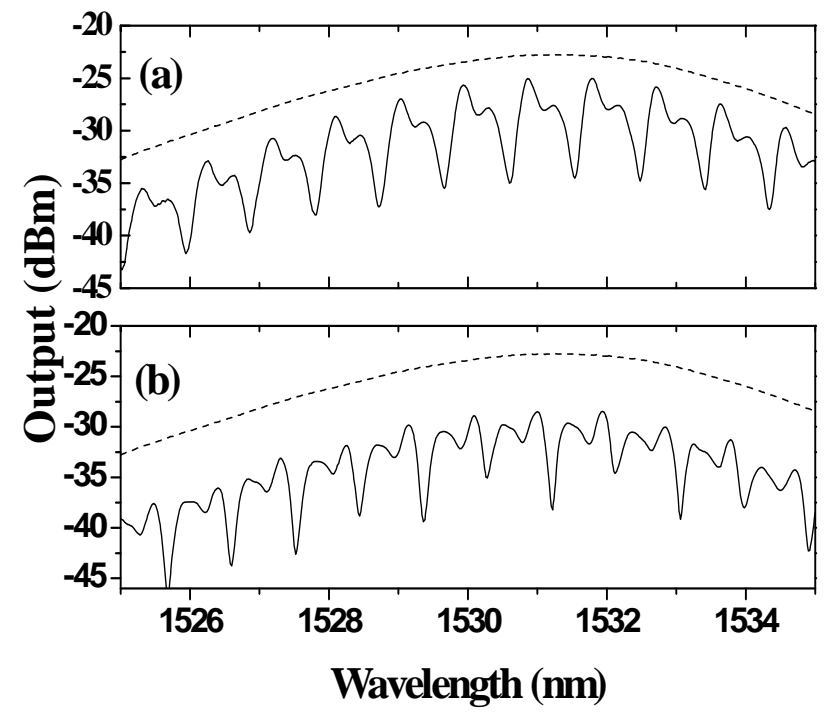

Fig. 3 Spectrum of MCR (a) three turns (b) four turns. The dashed line is the reference spectrum obtained for a free-standing microfiber.

The FSR of the three turn MCR was estimated with the simple ring resonator model. The resonating condition and FSR can be written as:

$$
\begin{gathered}
2 \pi n_{\text {eff }} L / \lambda=(2 m+1 / 2) \pi \\
\mathrm{FSR}=\frac{\lambda^{2}}{n_{\text {eff }} L}
\end{gathered}
$$

where $m$ is an integer, $\lambda$ the wavelength, $n_{\text {eff }}$ the effective index of the mode propagating in the microfiber and $L$ the loop length. $n_{\text {eff }}$ was calculated using the finite element method. This model predicts $\mathrm{FSR} 1 \mathrm{~nm}$ in good agreement with the experimental results. There are some differences because the microfiber and coil diameters present some irregularity and the microcoil position is not always perpendicular to the rod. With the aid of precisely controllable stages and rotation stages, it is possible to control the pitch and use thinner microfibers. If the rod is made of solvable materials, a high sensitivity microchannel sensor can also be simply obtained [10].

\section{Conclusion}

Microfiber coil resonators are manufacurated by wrapping a microfiber on a low refractive index rod. High extinction ratio and FSR have been obtained for three-turn and four-turns MCR.

The authors gratefully acknowledge initiating discussions with Profs. D.J. Richardson and J.S. Wilkinson, valuable interactions with $\mathrm{Dr}$. Neil Broderick, Dr. Katia Gallo and Dr. E. J. Tarbox, and financial support by the EPSRC, under the standard research grant EP/C00504X/1.

\section{References}

1. L. M. Tong et al, Nature, 426 (2003), 816-819.

2. G. Brambilla et al,Opt. Express, 12(2004), 22582263

3. S. G. Leon-Saval et al, Opt. Express,12 (2004), 2864-2869.

4. G. Brambilla et al, Electron. Lett. 42 (2006), 517519

5. C. Caspar et al, Electron. Lett., 25 (1989), 15061508.

6. M. Sumetsky et al, J. Lightwave Technol., 24 (2006), 242-249.

7. M. Sumetsky et al, Appl. Phys. Lett., 86 (2005), 161108.

8. X.Jiang et al,Appl. Phys. Lett., 88 (2006), 223501. 9. M. Sumetsky et al, Opt. Express, 13 (2004), 2303-2316

10. F. Xu et al, Opt. Express, 15 (2007),7888-7893,

11. M. Sumetsky, OFC (2007) PDP46.

12. G. Brambilla et al,Electron Lett. 41 (2005), 400401.

13. F.Xu et al, J. Lightwave Technol., (in press).

14. F. Xu et al,Appl. Opt., 46(2007), 570-573 